\section{WIDEBAND PLANAR MONOPOLE ANTENNAS FOR GPS/WLAN/WIMAX/ UWB AND X-BAND APPLICATIONS}

Goksenin Bozdag and Alp Kustepeli

Department of Electrical and Electronics Engineering, Izmir Institute of Technology, Izmir 35430, Turkey; Corresponding author: gokseninbozdag@iyte.edu.tr

Received 11 June 2015

ABSTRACT: In this article, two printed planar monopole antennas (PPMA) are presented. In the design of the first PPMA, the structure is divided into sections and they are optimized in the sense of bottom to up strategy. Tapered transitions and inset feed are employed to increase the bandwidth. The antenna operates between $2.37 \mathrm{GHz}$ and $12 \mathrm{GHz}$ with $V S W R<2$ and an average peak realized gain of $4.95 \mathrm{~dB}$. Therefore, it is suitable for WLAN, WiMAX, UWB, and X-Band applications. The second PPMA is designed by implementing slots on the previous one to include GPS. The resulting antenna operates in the 1.38-1.60 GHz and 2.33-13.74 GHz bands with VSWR $<2$. As a result, it also includes GPS in addition to the previous bands. The peak realized gain in GPS is $0.47 \mathrm{~dB}$ at $1.575 \mathrm{GHz}$ and the average peak realized gain is $4.41 \mathrm{~dB}$ for the 2.33$13.74 \mathrm{GHz}$ band. The group delay performances of the proposed PPMAs are also examined and the maximum group delay deviations of the first and the second PPMAs are observed as $1 \mathrm{~ns}$ and $1.33 \mathrm{~ns}$, respectively. (C) 2016 Wiley Periodicals, Inc. Microwave Opt Technol Lett 58:257-261, 2016; View this article online at wileyonlinelibrary.com. DOI 10.1002/mop.29550

Key words: printed planar monopole antennas; slotted antenna; wideband antennas

\section{INTRODUCTION}

The growth in wireless communication technologies has led to development of various wireless systems such as GPS, WLAN, WiMAX, and UWB. In addition to that, wireless systems have been commonly used for different purposes such as radar and biomedical applications in UWB and $\mathrm{X}$-band. As a result, today's space and weight limited, and low cost portable devices must be compatible with all of the above systems and hence they require a multiband or a wideband antenna [1-4].

A planar monopole antenna (PMA) is simply generated by replacing a monopole above the ground plane with a planar radiator sheet. Various geometries of planar monopoles have been proposed for many different applications since its first introduction by Dubost and Zisler [5] because PMAs provide wide bandwidth with very fine control and less degradation on the radiation pattern with an acceptable gain in the bandwidth [4,6]. In addition to the useful properties of PMAs, printed planar monopole antennas (PPMA) have other advantages such as small size, low weight, low cost, and ease of implementation. Moreover, low group delay deviation of PPMAs makes them suitable for pulse based systems such as UWB which has recently received great attention from industry. UWB provides more accuracy compared with the conventional systems especially for through-wall imaging, localization and biomedical diagnostics. UWB also offers very high data rate, multipath fading immunity and high resistance to jamming in short-range communication [7]. Therefore, in this study, it is intended to design and implement wideband PPMAs to cover all of the frequency bands mentioned above. Comprehensive numerical studies with parametric analysis are performed using HFSS to obtain the optimum designs before the fabrications and the measurements.

\section{ANTENNA DESIGNS, RESULTS, AND COMPARISONS}

Feeding line and radiating patch are the main components of a PPMA that need to be considered in the design of the antenna. Microstrip [8-13] and coplanar waveguide [14-18] structures are generally employed for feeding PPMAs. Even though various geometries have been used for the radiating patch in different applications $[11,12,15,17]$, tapered geometries are frequently used to obtain wide bandwidth, almost constant gain and group delay. Although there are also some studies proposing a general design procedure for specific type of PPMAs [19,20], there is no exact formulation in the literature and the designs are generally optimized by employing computational parametric studies. In this study, it is first intended to design a PPMA operating between $2 \mathrm{GHz}$ and $12 \mathrm{GHz}$ to cover WLAN, WiMAX, UWB, and X-Band. Microstrip feeding with partial ground is preferred because of its easy implementation and simpler design. Initially, a trapezoidal geometry is used as a radiating patch. Then, the dimensions of radiating patch, ground plane, and feeding line are determined as the main parameters to be optimized. Additionally, the feeding gap between ground plane and radiator patch is another critical parameter which must be taken into consideration $[4,15,19]$. Parametric studies are very useful to understand the effects of parameters on the performance of antennas. However, individual parametric studies are not enough to obtain desired antenna characteristics and all of the parameters must be optimized systematically. When the physical size constraints are taken into account, the maximum widths of the radiator patch and ground plane are chosen as to be equal to each other and the width $\left(W_{3}\right)$ is set to $35 \mathrm{~mm}$ as seen in Figure 1. The length of the ground plane $L_{\mathrm{g}}$ can be determined via the empirical formula

$$
L_{\mathrm{g}} \geq \frac{\lambda_{\max }}{5.35 \sqrt{\varepsilon_{\mathrm{r}}}}
$$

obtained by the comparisons of the computational and experimental results where $\lambda_{\max }$ is the maximum wavelength in the band. To make the total antenna length $L_{\mathrm{t}}$ as small as possible, $L_{\mathrm{g}}$ is chosen as $20 \mathrm{~mm}$, which corresponds to its minimum value to be used in GPS applications, as well. The width of the feeding line $\left(W_{\mathrm{f}}\right)$ is chosen as $3.56 \mathrm{~mm}$ to obtain a $50 \Omega$ characteristic line impedance. The other parameters are essentially optimized by means of bottom to up strategy [21]. Therefore, the antenna geometry shown in Figure 1(a) is divided into three design sections, two tapered transitions and a radiator, and the resulting antenna is called PPMA 1 . Two tapered transition sections provide smooth impedance transition between the feeding line and radiation section. The first tapered transition section, which is the one adjacent to the feed line, is the most sensitive part and therefore this section must be optimized first to get desired impedance bandwidth. The resulting widths and length of the first transition section are $W_{1}=4.06 \mathrm{~mm}, W_{2}=18 \mathrm{~mm}$, and $L_{1}=1.75 \mathrm{~mm}$, respectively. The second tapered transition section is employed to increase the bandwidth. The tapering angles of the two tapered sections are different. The widths of the second transition section are $W_{2}=18 \mathrm{~mm}$ and $W_{3}=35 \mathrm{~mm}$, and the length of that section is optimized as $L_{2}=18 \mathrm{~mm}$. The length of the radiation section has a significant effect on the minimum operating frequency of the antenna and the length of this section is optimized as $L_{3}=13 \mathrm{~mm}$ to obtain the desired frequency range. Additionally, the length of the feeding gap is optimized as $L_{\mathrm{fg}}=0.1 \mathrm{~mm}$ to improve impedance matching. $L_{\mathrm{fg}}$ and $L_{\mathrm{g}}$ are presented in Figure 1(c). Moreover, to obtain better 


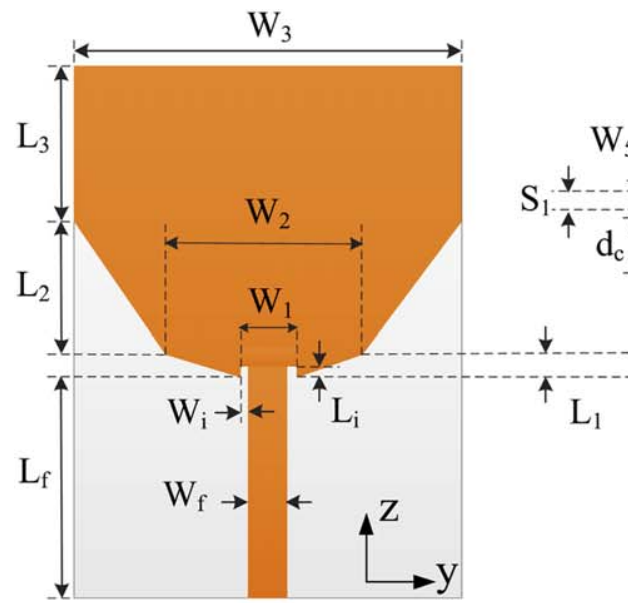

a)

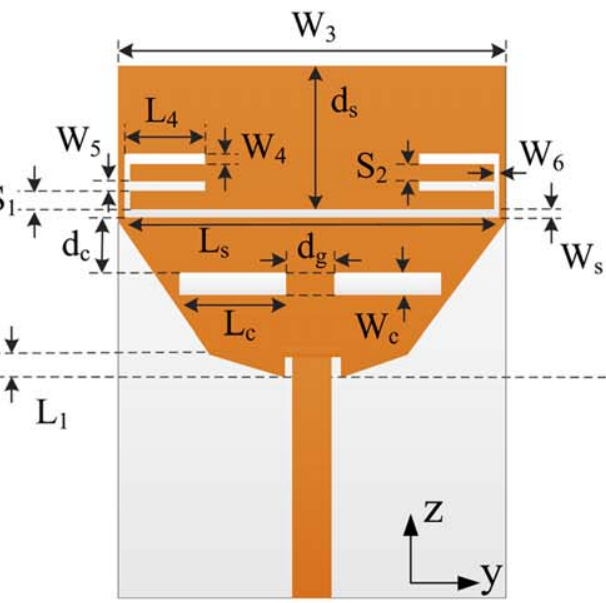

b)

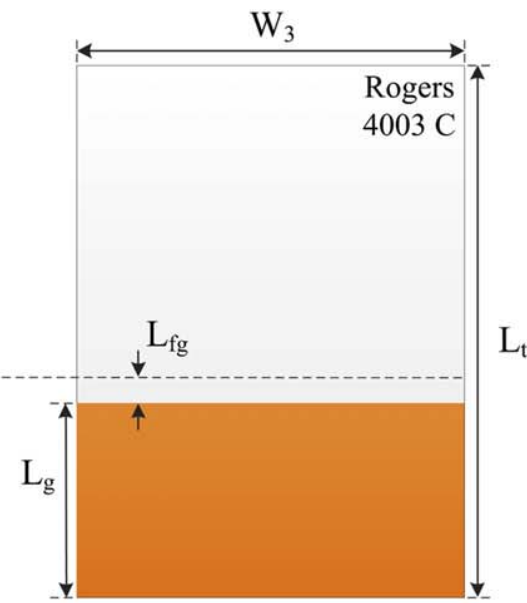

c)

Figure 1 Geometry of the PPMAs (a) front view of PPMA 1 with $W_{1}=4.06, L_{1}=1.75, W_{2}=18, L_{2}=13, W_{3}=35, L_{3}=13, W_{\mathrm{f}}=3.56, L_{\mathrm{f}}=20.1$, $W_{\mathrm{i}}=0.25, L_{\mathrm{i}}=0.75$, (b) front view of PPMA 2 with $W_{4}=0.75, L_{4}=7, W_{5}=0.75, W_{6}=0.5, W_{\mathrm{S}}=0.75, L_{\mathrm{S}}=34, W_{\mathrm{c}}=2, L_{\mathrm{c}}=10, S_{1}=1.75, S_{2}=1.5$, $d_{\mathrm{S}}=14.15, d_{\mathrm{c}}=4.25, d_{\mathrm{g}}=4.5$, and (c) back view of the PPMAs with $L_{\mathrm{g}}=20, L_{\mathrm{fg}}=0.1, \mathrm{~L}_{\mathrm{t}}=47.85$ (all dimensions are in mm.). [Color figure can be viewed in the online issue, which is available at wileyonlinelibrary.com]

impedance matching, an inset is also employed and its length and width are optimized as $L_{\mathrm{i}}=0.75 \mathrm{~mm}$ and $W_{\mathrm{i}}=0.25 \mathrm{~mm}$, respectively. Finally, the width and the length of the antenna are obtained as $35 \mathrm{~mm}$ and $47.85 \mathrm{~mm}$, respectively. The antennas were fabricated using photolithography and commercially available substrate Rogers $4003 \mathrm{C}$ whose relative dielectric constant $\left(\varepsilon_{\mathrm{r}}\right)$, tangent loss and thickness are 3.55, 0.0014, and $1.524 \mathrm{~mm}$, respectively. The fabricated PPMA 1 is shown in Figure 2(a). The computed and measured VSWR results of the antenna are presented in Figure 3. As seen from the figure, the computed operating bandwidth is between 2 and $12.5 \mathrm{GHz}$, and the measured one is between 2.37 and $12 \mathrm{GHz}$ (5:1) which covers WLAN, WiMAX, UWB, and X-Band.

Implementation of proper slots having specific locations on an antenna is generally used for the excitation of additional operating frequencies $[14,18]$, band stopping $[10,12,15-17,19]$, antenna miniaturization [9], gain, and bandwidth enhancements $[8,11,13]$. Therefore, slots are employed on PPMA 1 to include GPS, as well, and the resulting antenna is called PPMA 2. Its

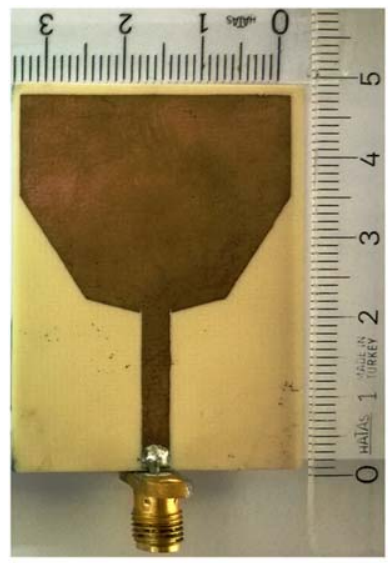

(a)

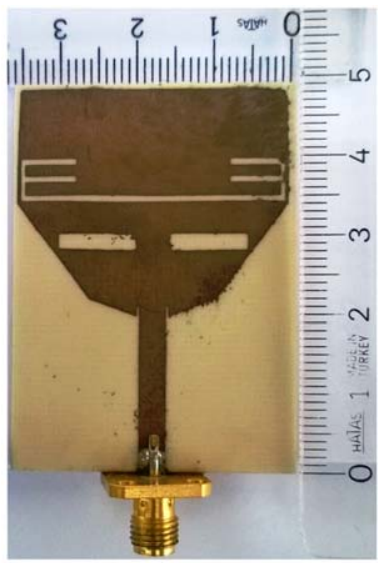

(b)
Figure 2 Fabricated PPMAs (a) PPMA 1 and (b) PPMA 2. [Color figure can be viewed in the online issue, which is available at wileyonlinelibrary. com] front and back views are presented in Figures 1(b) and 1(c), respectively. GPS band is basically included using the upper slot shown in Figure 1(b). Since the edge of the transition sections are more sensitive, the slot is located closer to the edge of the second transition section, and its geometry and position are

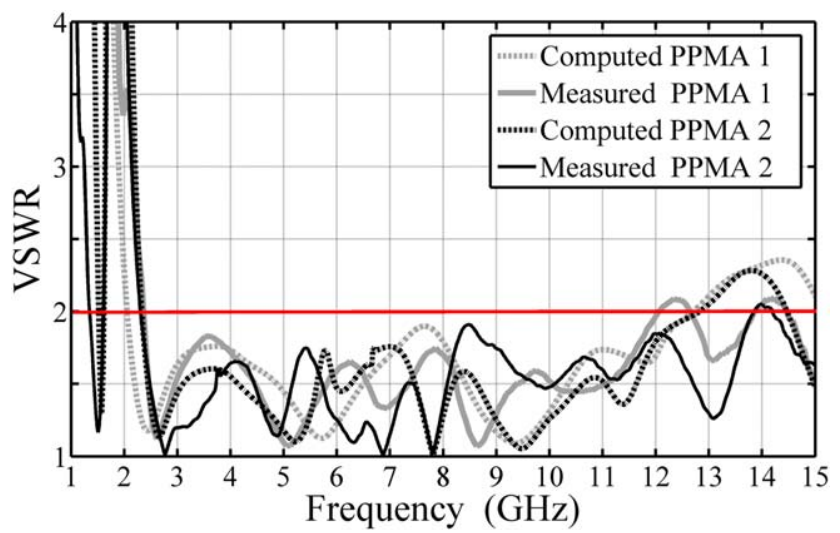

Figure 3 VSWRs of PPMAs. [Color figure can be viewed in the online issue, which is available at wileyonlinelibrary.com]

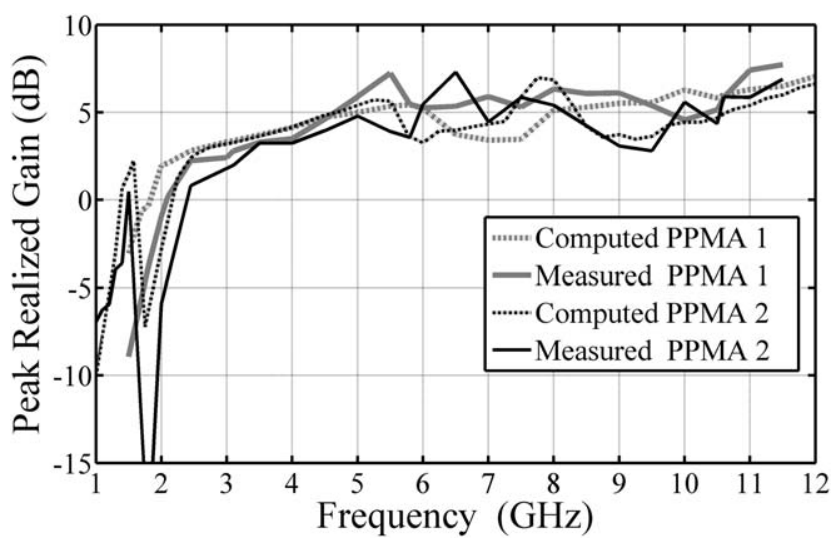

Figure 4 Peak realized gains of PPMAs. [Color figure can be viewed in the online issue, which is available at wileyonlinelibrary.com] 


\section{$x-z$ plane}
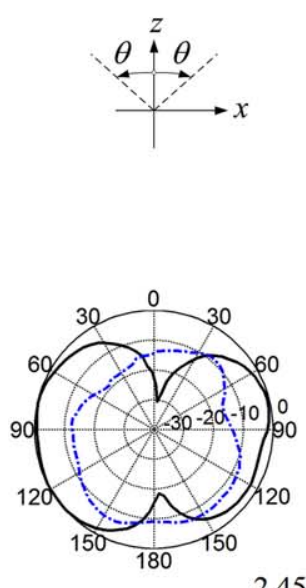

$2.45 \mathrm{GHz}$

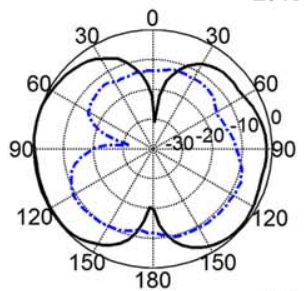

$3.1 \mathrm{GHz}$
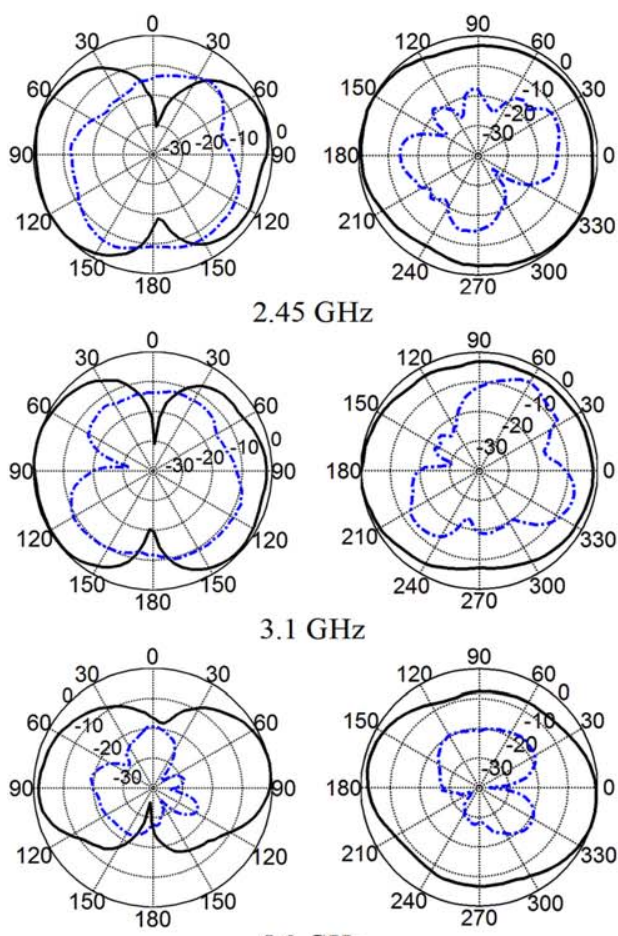

$x-y$ plane
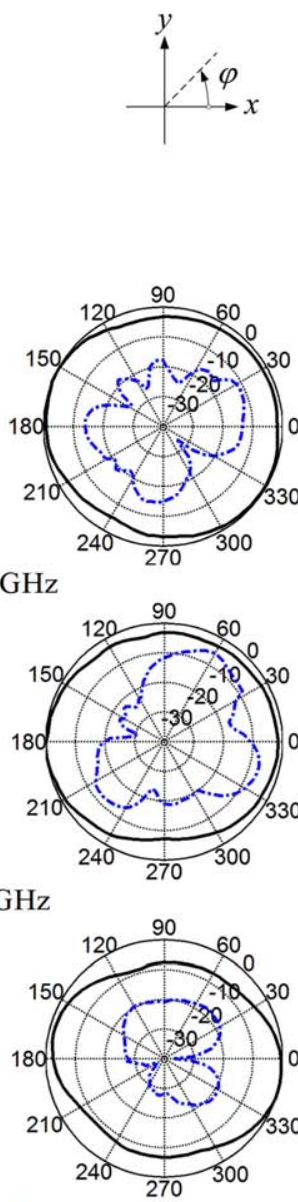

$5.8 \mathrm{GHz}$
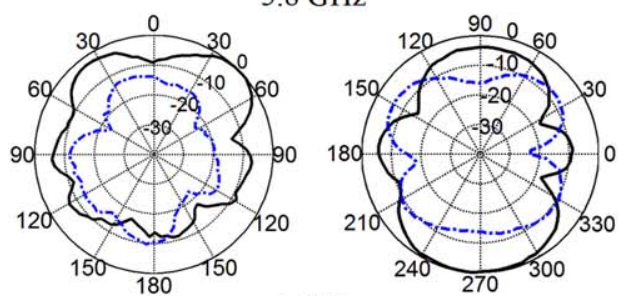

$8 \mathrm{GHz}$
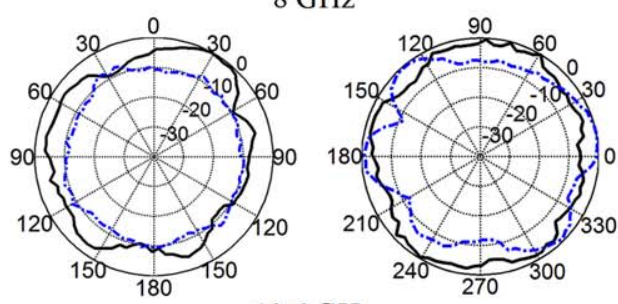

$10.6 \mathrm{GHz}$

(a)
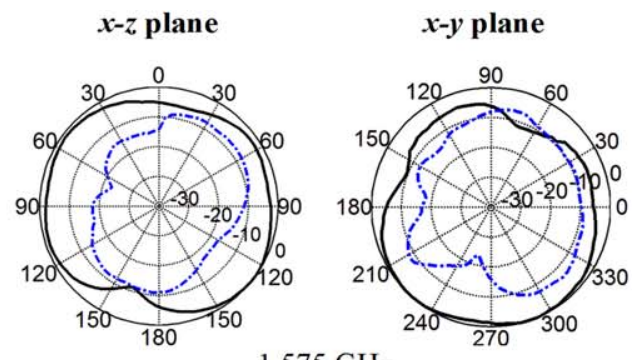

$1.575 \mathrm{GHz}$
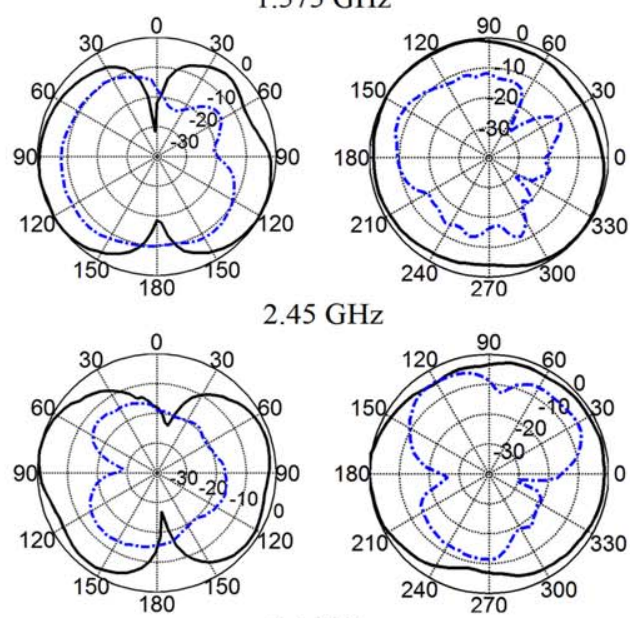

$3.1 \mathrm{GHz}$
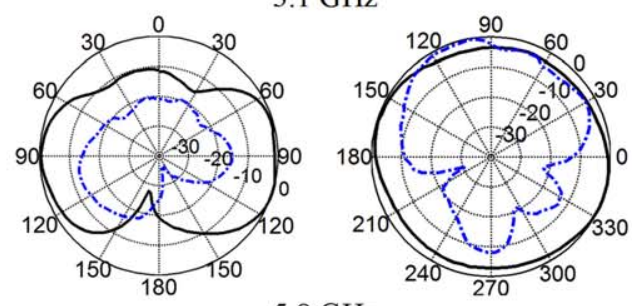

$5.8 \mathrm{GHz}$
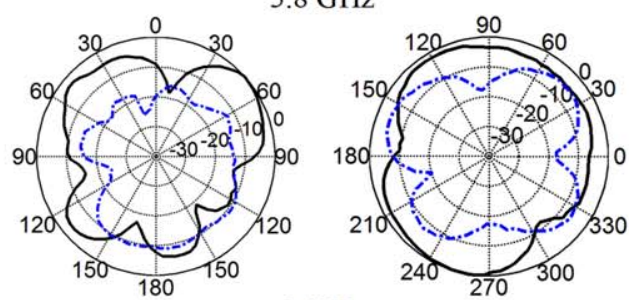

$8 \mathrm{GHz}$
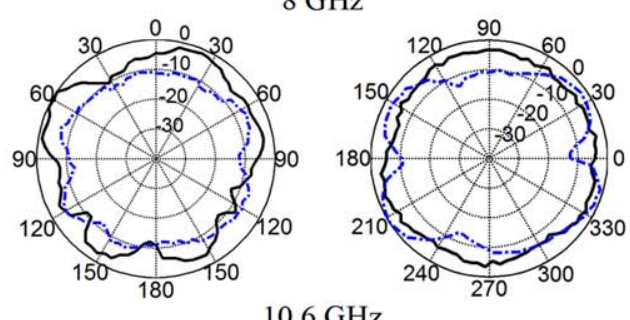

$10.6 \mathrm{GHz}$

(b)

Figure 5 Measured normalized radiation patterns of PPMAs (a) PPMA 1 (b) PPMA 2. [Color figure can be viewed in the online issue, which is available at wileyonlinelibrary.com]

also optimized. In addition to that, the length of the radiator section $\left(L_{3}\right)$ is increased by $1.15 \mathrm{~mm}$ to obtain the desired frequency for GPS. Conversely, these modifications deteriorate the impedance matching at higher frequencies and many fluctuations are observed. To overcome this problem, the length of the inset feed $\left(L_{\mathrm{i}}\right)$ is increased to $1.5 \mathrm{~mm}$ and compensation slots [8], located symmetrically on the second transition section, are implemented as seen in Figure 1(b). As the fluctuations occur at higher frequencies with narrow bandwidths, the lengths of those slots are smaller. The fabricated PPMA 2 is shown in Figure 2(b). The computed and measured VSWR results of PPMA 2 are also presented in Figure 3. According to the computed 


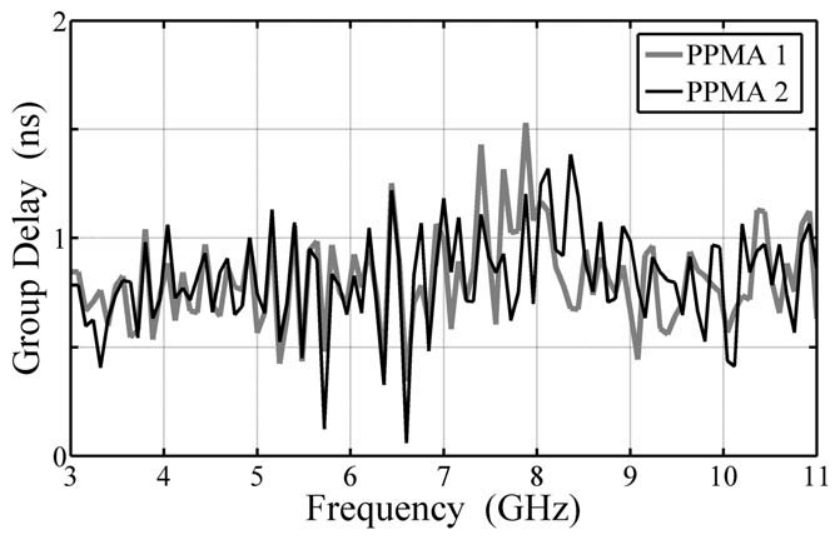

Figure 6 Measured group delays of PPMAs

results, it is observed that the two operating bands are 1.52$1.61 \mathrm{GHz}$ and $2.35-13.43 \mathrm{GHz}$. In the measured results, it is seen that the bands are $1.38-1.60 \mathrm{GHz}$ and $2.33-13.74 \mathrm{GHz}$ (5.9:1), which make the antenna suitable for GPS $\left(L_{1}, L_{3}\right.$, and $L_{4}$ ), WLAN, WiMAX, UWB, and X-Band. The computed and measured results are in good agreement. The performances of the designed PPMAs are examined in terms of realized gain, radiation pattern and group delay, as well. The antenna gains used in the calculation of measured results were obtained by the two antenna method presented in [22]. The computed and measured peak realized gains $\left(G_{\mathrm{pr}}\right)$ of the proposed PPMAs are given in Figure 4. According to the figure, the maximum value of the measured $G_{\mathrm{pr}}$ of PPMA 1 reaches $7.72 \mathrm{~dB}$ at $11.5 \mathrm{GHz}$ and its average value is $4.95 \mathrm{~dB}$ in the operating band. The maximum value of the measured $G_{\mathrm{pr}}$ of PPMA 2 is $7.29 \mathrm{~dB}$ at $6.5 \mathrm{GHz}$. At $1.575 \mathrm{GHz}$, which represents GPS, the measured $G_{\mathrm{pr}}$ is 0.47 $\mathrm{dB}$ and the average measured $G_{\mathrm{pr}}$ is $4.41 \mathrm{~dB}$ in the 2.33$13.74 \mathrm{GHz}$ band. It is seen from the figure that the computed and measured peak realized gains are in good agreement. The measured normalized copolarization and cross-polarization patterns of the proposed PPMAs are presented in Figure 5. It is observed from the figure that the radiation patterns of PPMAs, especially the ones for PPMA 2, are almost omnidirectional in $x-y$ plane. One can also see that cross polarization discrimination levels are $10 \mathrm{~dB}$ or higher in the $x$ direction at low frequencies but these levels decrease at high frequencies. Group delay $\tau_{g}$ is another parameter which is used to examine the time delay of signal while transmitting through a device. It is given by $\tau_{g}=$ $-d \varnothing / d \omega$ where $\varnothing$ is the phase response of signal and $\omega$ is angular frequency. Although constant group delay is desired to avoid signal distortions for transmission, it is not generally possible in applications and small deviations are acceptable. The measured group delay performances of the proposed antennas are presented in Figure 6. As the group delay performance of the antenna has a critical role especially in impulse based communication applications, measurements were made for only UWB. Group delay was obtained using the method given in [23] and the antennas were placed $20 \mathrm{~cm}$ away from each other. As can be seen from Figure 6, PPMA 1 and PPMA 2 have maximum group delay deviations of 1 and $1.33 \mathrm{~ns}$, respectively. It can be said that the group delay performances of the antennas are good for UWB applications.

\section{CONCLUSION}

In this study, two PPMAs were designed and realized. Bottom to up strategy was employed for the design of the first PPMA and it was divided into sections which were optimized to obtain broader bandwidth. The resulting antenna operates in the 2.37 $12 \mathrm{GHz}$ band with an average peak realized gain of $4.95 \mathrm{~dB}$. The antenna is suitable for WLAN, WiMAX, UWB, and XBand applications. The second PPMA was designed by implementing slots on the first design. It is the improved version of the first one and it operates in the $1.38-1.60 \mathrm{GHz}$ band, where GPS $\left(L_{1}, L_{3}\right.$, and $\left.L_{4}\right)$ is allocated, and the $2.38-13.74 \mathrm{GHz}$ band, where WLAN, WiMAX, UWB, and X-Band are allocated. The measured group delays of the first and the second PPMAs are satisfactory with maximum $1 \mathrm{~ns}$ and $1.33 \mathrm{~ns}$ deviations in the whole UWB, respectively. As a result, these two antennas are good candidates for wideband, multipurpose and portable devices.

\section{REFERENCES}

1. P. Vainikainen, J. Holopainen, C. Icheln, O. Kivekas, M. Kyro, M. Mustonen, S. Ranvier, R. Valkonen, and J. Villanen, More than 20 antenna elements in future mobile phones, threat or opportunity?, In: 3rd European Conference on Antennas and Propagation, Berlin, Germany, 2009, pp. 2940-2943.

2. A. Asghar, M. Malick, M. Karlsson, and A. Hussain, A multiwideband planar monopole antenna for $4 \mathrm{~g}$ devices, Microwave Opt Technol Lett 55 (2013), 589-593.

3. S. Praveen, O. Raoul, P. Bradley, A. David, L. Todd, S. Daniel, K. Jonathan, and P. John, Miniature radar for mobile devices, In: High Performance Extreme Computing Conference, Waltham, MA, 2013, pp. 1-8.

4. M. Ammann, Control of the impedance bandwidth of wideband planar monopole antennas using a beveling technique, Microwave Opt Technol Lett 30 (2001), 229-232.

5. G. Dubost and S. Zisler, Antennas a large bande, Masson, Paris 1976.

6. N.P. Agrawall, G. Kumar, and K. Ray,Wide-band planar monopole antennas, IEEE Trans Antennas Propag 46 (1998), 294-295.

7. M.Z. Win, D. Dardari, A.F. Molisch, W. Wiesbeck, and J. Zhang, History and applications of UWB, In: Proceedings of IEEE, 2009, pp. 198-204.

8. Z. Low, J. Cheong, and C. Law, Low-cost pcb antenna for uwb applications, IEEE Antennas Wireless Propag Lett 4 (2005), 237-239.

9. Y. Lu, Y. Huang, H.T. Chattha, and Y. Shen,Technique for minimising the effects of ground plane on planar ultra-wideband monopole antennas, IET Microwaves Antennas Propag 6 (2012), 510-518.

10. M. Ojaroudi and N. Ojaroudi, Ultra-wideband slot antenna with frequency band-stop operation, Microwave Opt Technol. Lett. 55 (2013), 2020-2023.

11. K. Ray, S. Thakur, and A. Deshmukh, Slot cut printed elliptical UWB monopole antenna, Microwave Opt Technol Lett 56 (2014), 631-635.

12. S.S. Mirmosaei, S.E. Afjei, E. Mehrshahi, and M.M. Fakharian, A dual band-notched ultra-wideband monopole antenna with spiralslots and folded SIR-DGS as notch band structures, Int J Microwave Wireless Technol (2015), DOI: 10.1017/S1759078715000719.

13. P. Beigi, J. Nourinia, B. Mohammadi, and A. Valizafe, Bandwidth enhancement of small square monopole antenna with dual band notch characteristic using U-shaped slot and butterfly shape parasitic element on backplane for UWB applications, Aces J 30 (2015), 78-85.

14. J.H. Kim, J.N. Lee, and J.K. Park,Triple-band antenna with slots for PCS/WLAN/UWB applications, Microwave Opt Technol Lett 52 (2010), 2540-2544.

15. D. Parkash and R. Khanna, Triple band rectangular-shaped monopole antenna for WLAN/WiMAX/UWB applications, Microwave Opt Technol Lett 56 (2014), 2113-2117.

16. Y. Zehforoosh and T. Sedghi, A cpw-fed printed antenna with bandnotched function using an m-shaped slot, Microwave Opt Technol Lett 56 (2014), 1088-1092.

17. M. Jagannath, P.C. Kalaria, and M.V. Kartikeyan, Transient response of dual-band notched ultra-wideband antenna, Int J Microwave Wireless Technol 7 (2015), 61-67. 
18. R. Wu, P. Wang, Q. Zheng, and R. Li, Compact CPW-fed triple band antenna for diversity applications, Elect Lett 56 (2015), 735-736.

19. J. Wang and X. He, Analysis and design of a novel compact multiband printed monopole antenna, Int J Antennas Propag 2013 (2013), Article ID 694819

20. K.P. Ray and S. Thakur, Ultra wide band vertex truncated printed pentagon monopole antenna, Microwave Opt Technol Lett 56 (2014), 2228-2234.

21. D. Tran, On the design of a super wideband antenna, ultra wideband, In: B. Lembrikov (Ed.), ISBN 978-953-307-139-8, InTech, DOI 10.5772/10000 2010. Available at: http://www.intechopen.com/ books/ultra-wideband/on-the-design-of-a-super-wideband-antenna, 2010.

22. C.A. Balanis, Antenna theory: Analysis and design, Wiley, Hoboken, NJ, 2012.

23. P. McEvoy, M. John, S. Curto, and M.J. Ammann, Group delay performance of ultra wideband monopole antennas for communication applications, In: Antennas and Propagation Conference, Loughborough, UK, 2008, pp. 377-380.

(C) 2016 Wiley Periodicals, Inc.

\section{SURFACE PLASMON WAVE APPLICATION IN MICROWAVE MODULATOR INDUCED BY SWITCHABLE METAMATERIAL}

\author{
Wenjuan Zhang, Liguo Sun, Fujiang Lin, Guiqiang Zhu, and \\ Diqun Lu \\ Micro/Nano Electronics System Integration Research Centre, \\ University of Science and Technology of China, Hefei 230027 \\ People's Republic of China; Corresponding author: \\ liguos@ustc.edu.cn
}

Received 12 June 2015

ABSTRACT: Corrugated metal surface with underlayer metal ground can propagate surface waves when the operation frequency is below the asymptotic limit-the surface plasma frequency. Tunability of the spoof surface plasmon polariton (SSPP) wave is realized by placing metamaterial underneath the SSPP strip. We design and experimentally demonstrate a modulator based on the planar surface plasmon waveguide and switchable metamaterial, where PIN diodes are implemented to tune the metamaterial. With the diodes working in on/off states, the proposed modulator can be, respectively, viewed as a signal-stop/signal-pass circuit at the operation frequency. The experimental results validated this method. It is observed that the proposed modulator would operate from 2.51 to $2.57 \mathrm{GHz}$ with $12 \mathrm{~dB}$ on-off isolation. (C) 2016 Wiley Periodicals, Inc. Microwave Opt Technol Lett 58:261-263, 2016; View this article online at wileyonlinelibrary.com. DOI 10.1002/mop. 29549

Key words: amplitude shift keying; spoof surface plasmon polariton; metamaterial; microwave-wave switch; modulator; PIN diode

\section{INTRODUCTION}

Surface plasmon polariton (SPP) is surface wave that propagates on the surface of a conductor. Plasmonic metamaterials have been proposed to support surface waves at the terahertz and microwave frequencies, which have similar dispersion diagram to the SPPs at the visible frequencies. They are termed as spoof SPPs (SSPPs) [1]. Among them, ultrathin corrugated metallic strip structure is very attractive and reported recently [2-5]. The approach to achieve conversion from guided wave to SSPPs and its wave manipulation has been well studied. This unusual property may be exploited to realize microwave device.

Metamaterial is an attractive area with great many contributions in the general applications such as perfect absorbers. Typi- cally, metamaterial consists of patterned metallic inclusions within a dielectric medium. Switchable resonant metamaterial means the resonance frequency of metamaterial structures can be tuned by an external stimulus. Several methods have been reported to make metamaterial switchable. A common solution for this is to tune the metamaterial performance by embedding diodes as controllable devices [6].

In this letter, a surface plasmon wave based modulator is proposed, SSPP strip along with PIN diode switchable metamaterial will form the modulation function. By applying a tuning voltage bias for PIN diodes, the coupling between the metamaterial and the SSPP strip will disturb the surface plasmon wave propagation, thus leading to signal-stop or signal-pass effect at the operation frequency. It is observed that the proposed modulator would operate from 2.51 to $2.57 \mathrm{GHz}$ with $12 \mathrm{~dB}$ on-off isolation. This work will pave the way for SSPP application in microwave device.

\section{MELIORATED SPOOF SPP WAVEGUIDE}

At first, we briefly introduce a corrugated metallic strip on which spoof SPP mode is supported [3]. Figure 1(a) illustrates the schematic configuration of the high-efficiency SSPP. The structure consists of two parts: conversion and matching section (part I), and a SSPP strip (part II). Figure 1(b) shows that the wave vector is deviating from the light line and is asymptotically approach to a cutoff frequency, thus indicates that the periodic structure with underlayer metal plane as ground is capable of confining EM waves on the surface.

\section{DETAILED MODULATOR DESIGN AND FABRICATION}

As stated in the work [3], the SSPP wave propagation can be manipulated by adding metamaterial below the SSPP strip. Electrically controlled metamaterial can be realized using diodes embedded between adjacent resonant units. This permits us to design an electrically controlled OOK modulator.

Figure 2(a)-2(b) is the fabricated modulator. Figure 2(c) 2(e) details the structure. In Figure 2(d), the dashed lines help mark the location of the $3 \times 4$ metamaterial array. We adopt the electrically coupled LC resonators [7] based metamaterial.

The SSPP strip is on the top of the dielectric substrate. The metamaterial array on the second layer is aligned symmetrically under the SSPP strip. The dimension of one unit cell is shown in Figure 2(e). The third layer is for the ground plane. Diodes are placed on the bottom layer, and they are connected to the metamaterial through vias. A pair of control lines is soldered on the bottom layer.

The first and forth copper layers are of $0.035 \mathrm{~mm}$ thickness. The second and third copper layers are of $0.0175 \mathrm{~mm}$. The dielectric is Rogers 4350. Vertically the SSPP strip is separated from the metamaterial array by $0.508 \mathrm{~mm}$, the metamaterial array from the ground by $0.2 \mathrm{~mm}$, and the ground from the feeding lines by $0.508 \mathrm{~mm}$.

As shown in Figure 2, PIN diodes (SMP1320-079LF) are selected due to its good switch performance at microwave frequency. Diodes are placed in series between the neighboring unit cells within each column. When the diodes are turned on (off), they electrically short (open) all the unit cells in the column. The diodes on-off states support different electromagnetic modes in the metamaterial resulting in SSPP wave rejection or propagation at certain frequency band. In order to turn on the diodes, a DC bias voltage, exceeding the sum of threshold voltage of diodes in one column, is applied across the "+" and "-" lines in Figure 2. 